\title{
Compartmental analysis and model building in the study of glucose kinetics in the lactating cow
}

\author{
By S. Horsfield, Jennifer M. Infield and E. F. Annison, Unilever Research \\ Laboratory, Colworth House, Sharnbrook, Bedford
}

The kinetics of substrate transfer and turnover in many biological systems may be modelled using the concept of compartments with interconnecting pathways. Linear compartmental models in which substrate turnover is constant have been widely studied and subjected to rigorous mathematical analysis (Berman \& Schoenfield, 1956; Steele, Wall, de Bodo \& Altszuler, i 956; Steele, 1964). These studies have largely stemmed from the use of isotopic tracers introduced into the system as a single injection or continuous infusion, or by a combination of both techniques. Irrespective of the mode of tracer introduction, the resultant radioactivity in any one compartment may be represented by a function involving a sum of exponentials, with the number of exponentials equal to the number of compartments of the theoretical model.

When data are available for all of the compartments, the parameters of the general model (Fig. I) can be uniquely determined (Berman \& Schoenfield, 1956). Skinner, Clark, Baker \& Shipley (1959) have produced the complete solution to the general linear three-compartment model (Fig. I) after a single injection of isotope. The method is based on fitting the sum of three exponentials to data from each compartment and then using the intercepts and exponents in a series of algebraic equations to obtain the solution. For incomplete data some of the rate constants have to be fixed, normally equal to zero, to obtain a solution.

Frequently, it is not possible to collect data on all the compartments and the general model is indeterminate. The analysis of incomplete data, the usual situation in isotope dilution studies with whole animals where data are available only for the injected pool, has been mainly concerned with estimating three kinetic variables: the net rate of transport of substrate through the system (irreversible disposal rate), the mass of the injected compartment (pool size) and the rate at which substrate enters this compartment (total entry rate). The calculation of these variables requires the fitting of a sum of exponentials, and the intercepts and exponents of the exponentials are combined to estimate irreversible disposal rate, total entry rate and pool size (see Leng, 1970).

These methods of analysis of incomplete data have serious limitations. Julius (1972) has indicated that the sum of exponentials fit is frequently insensitive to appropriately chosen changes in the exponents and intercepts. This casts some doubt on the validity of fitting a sum of exponentials as a basis for data analysis, 


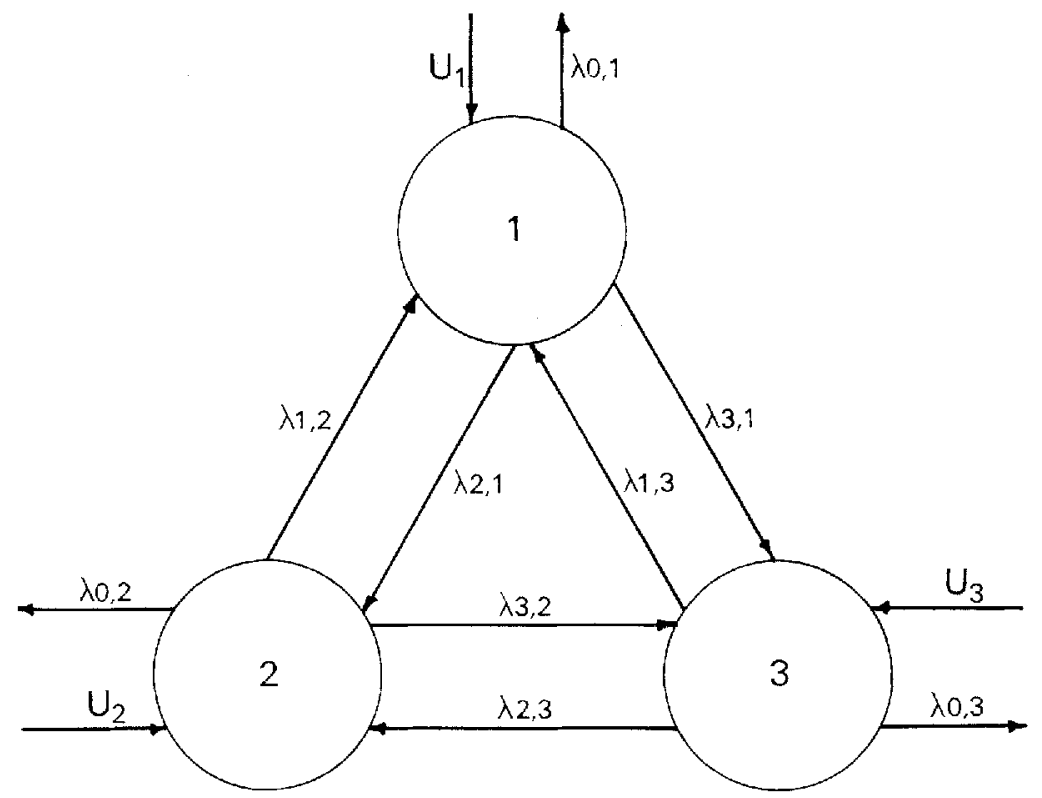

Fig. $I$. The general three-compartment model with inputs $U_{1}, U_{2}$ and $U_{3}$, and rate constants $(\lambda i j$, representing the fractional turnover of substrate flowing from compartment $j$ to compartment $i$ ).

and for certain sets of ill-conditioned data there may be serious errors in the estimated kinetic parameters. A further limitation is that prior estimates of rate constants and compartment sizes from other experiments cannot easily be incorporated into these methods of analysis.

An alternative approach to the multi-exponential analysis of data obtained by isotope dilution studies in whole animals is to analyse the data in terms of models which fit the data and are consistent with other relevant physiological information.

The kinetics of any compartmental model may be expressed mathematically in terms of a set of simultaneous differential equations. For example, the differential equations defining the kinetics of the isotope in a general linear compartment model are:

$$
\frac{\mathrm{dq}_{\mathrm{i}}(\mathrm{t})}{\mathrm{dt}}=-\left(\begin{array}{l}
\mathrm{n} \\
\sum_{\substack{\mathrm{j}=0 \\
\mathrm{j} \neq \mathrm{i}}} \lambda_{\mathrm{i}} \\
\mathrm{j}
\end{array}\right) \quad \mathrm{q}_{\mathrm{i}}(\mathrm{t})+\sum_{\substack{\mathrm{j}=\mathrm{r} \\
\mathrm{j} \neq \mathrm{i}}}^{\mathrm{n}} \mathrm{q}_{\mathrm{j}}(\mathrm{t})+\mathrm{U}_{\mathrm{i}}(\mathrm{t})(\mathrm{i}=\mathbf{I}, 2 \ldots \mathrm{n})
$$

Where $q_{i}(t)$ - The amount of labelled material in the $i^{\text {th }}$ compartment at time $t$ $(\mathrm{i}=\mathrm{I}, \ldots, \mathrm{n})$.

$\lambda_{\mathrm{oi}}$ - The fractional amount of material leaving the system from $i^{\text {th }}$ compartment per unit time $(i=I, \ldots, n)$. 
$\lambda_{1 j}-$ The fractional amount of material in the $\mathrm{j}^{\text {th }}$ compartment which moves to the $i^{\text {th }}$ compartment per unit time $(i=I, \ldots, n ; j=r$, $\ldots, n ; j \neq i)$.

$\mathrm{U}_{1}(\mathrm{t})$ - An input function of labelled material into the $\mathrm{i}^{\text {th }}$ compartment $(i=I, \ldots, n)$. For single shot experiments $U_{i}(t)=0$ for all $i$.

Similar sets of differential equations can be defined for other models including non-linear compartment models. This general mathematical description of compartmental models form the basis of the SAAM 25 (Simulation, Analysis and Modelling) computer programme, written and developed by Berman \& Weiss (1967). The differential equations which define the model form part of the input to the programme. The model parameters may be fixed (normally equal to zero) or estimated within the programme. Parameters which are to be estimated require initial estimates and upper and lower limits within which the solution will lie. A steady-state solution can be obtained for the compartment sizes and flow rates between the compartments of unlabelled substrate. These values can indicate the correspondence between the biological system and the compartmental model. The data analysed by the programme usually take the form of observed specific radioactivities together with their standard errors over a given time interval for each compartment. If only incomplete data are available, some of the model parameters have to be fixed to obtain a unique solution. Berman \& Schoenfield (r956) indicated that for an $n$-compartment model there are, in general, only $2 n-$ I estimable parameters if only a single compartment is available for measurement. Measurement on each additional compartment gives at most $n-I$ extra estimable parameters.

Estimates for the model parameters, compartment sizes and steady-state inputs and flow rates, or any function of these parameters, can be entered as additional data together with their corresponding standard errors. This facility enables results from other experiments, possibly not tracer experiments, to be combined in the analysis in order to reduce the indeterminancy of the model, particularly when the data are incomplete. Each item of data is weighted, in inverse proportion to its squared standard error, for statistical inclusion in the solution.

For a given set of model parameters, the programme solves the differential equations to obtain calculated estimates of the amounts in each compartment at points in time corresponding to the observed data and estimates of any functions or parameters entered as data. A weighted sum of squared differences between the observed data and the calculated data is obtained. The programme then tries to minimise this weighted sum of squares by adjusting the variable model parameters within their upper and lower limits. A revised set of model parameters is obtained and the whole process is repeated until the weighted sum of squares is minimised.

The set of model parameters then constitute a solution to the model which has to be checked for convergence and uniqueness. If there are systematic deviations between the calculated and the observed data, then either too many of the model parameters have been fixed or the wrong ones have been fixed, and in either situation the 
model is incorrect. If the standard errors associated with the calculated parameters are large in comparison with the parameters' values, then the data are insufficient to determine the model. If neither of the above conditions occurs, the solution is said to have converged and is unique, and the model parameters constitute the least squares solution of the model. Berman ( $\left.196_{3}\right)$ has indicated how to proceed if either of the above conditions occurs. The routine output of a model solution gives all the parameter values and, if requested, a steady-state solution for compartment sizes and flow rates. The standard errors of all the calculated values are obtained. The programme thus provides a flexible approach to the analysis of compartmental models allowing a number of model configurations to be analysed and more detailed interpretation of the data made.

Kronfeld, Ramberg \& Shames (I97I) discussed at length the application of the SAAM 25 programme to the compartmental analysis of glucose kinetics in the cow. We have adopted the same procedures in extending the general approach of Kronfeld et al. (1971) to lactating cows of defined energy status. Metabolizable energy (ME) intakes were either calculated from the level and composition of the diet, or in some instances were measured in animals housed in respiration chambers (Infield, Robb, Horsfield \& Annison, 1974). The cows were prepared with an exteriorized carotid artery and mammary vein (Bickerstaffe, Annison \& Linzell, 1974), and were fed either hay or grass silage with a commercial concentrate to maintain a positive energy balance.

$\left[\mathrm{U}-{ }^{14} \mathrm{C}\right]$ glucose $(2 \mathrm{mCi})$ was injected into the jugular vein of fed cows, and arterial blood samples $(5 \mathrm{ml})$ were withdrawn via a catheter from the carotid artery at intervals for $24 \mathrm{~h}$ for the measurement of glucose specific radioactivity without disturbing the normal feeding and milking of the animal. The data were analysed by multi-exponential analysis to obtain values for glucose irreversible loss, total entry rate and pool size (Table I), and also used for model building and compartmental analysis. A four-compartment model (Fig. 2) best fitted the data. This model is an extension of the three-compartment model of Kronfeld et al. (1971), and embodies the same general features. The results from twelve experiments were fitted to the four-compartment model. Values for the rate constants representing the fractional turnover between compartments $\left(\lambda_{i j}\right)$, steady-state compartment sizes for glucose (or products-precursors) $\left(Q_{i}\right)$ and the flow rates of glucose between compartments $\left(\mathrm{R}_{\mathrm{ij}}\right)$ are shown for four experiments (Table 2). Pool I accounted for about $1 \mathrm{I} \%$ of body-weight, and the sum of compartments I and 2 about $18 \%$ (Table I). The latter value may represent extracellular space, but is somewhat lower than the value obtained by Kronfeld et al. (1971). Compartments 3 and 4 may represent two groups of products-precursors which turn over at different rates, i.e. compartment 3 might include lactate, glycerol and glucogenic amino acids, and compartment 4 glycogen, ribose-deoxyribose and mucopolysaccharides.

The flow of glucose out of the system, $R_{0,3}$, which is identical to the glucose inflow, may be equated with glucose irreversible loss determined by multi-exponential analysis. Similarly, $\mathrm{R}_{2,1}$ corresponds to total entry rate, and good agreement was obtained between the parameters determined by multi-exponential analysis and from 


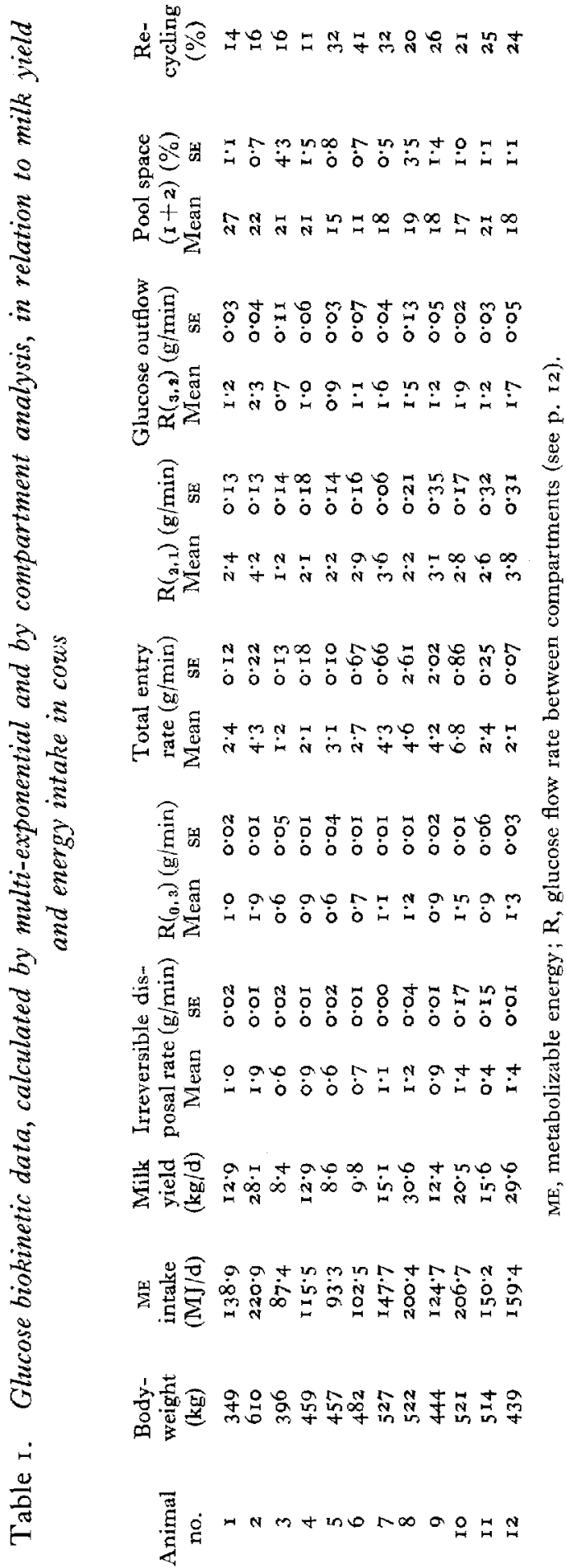


the model. (Table $I$ ). A highly significant relationship was observed between $R_{0,3}(y)$ and $M E$ intake ( $\mathrm{x}$ ), as shown by the regression equations derived from weighted least squares analysis $(y=0.013+0.029 x, r=0.90, P<0.001)$. Judson \& Leng ( 1968 ) showed that glucose entry rate was closely correlated with digestible energy intake in sheep. Glucose outflow $R_{0,3}$ was also correlated with milk yield $\left(Y_{1}\right)\left(Y_{1}=\right.$ $0.52+0.029 x, r=0.74, P<0.005)$, and to lactose output $\left(\mathrm{Y}_{2}\right)\left(\mathrm{Y}_{2}=0.47+0.94 \mathrm{x}\right.$, $r=0.84, \mathrm{P}<0.00 \mathrm{I})$. No correlation was observed between total entry rate, or $\mathrm{R}_{\mathbf{2}, 1}$, and $M E$ intake or milk yield. Glucose recycling represented by the glucose flow $R_{1,3}$ is shown in Table $I$ as a percentage of $R_{3,2}$, since from the model we may equate the latter flow with the movement of glucose from extracellular space. The extent of recycling was not correlated with ME intake or milk yield.

The proposed model can be no more than a rough approximation of the multicompartmental system that comprises the whole animal. Although the data fit the model, the present model must be regarded as a degenerate case of one of higher order. A major limitation of the present approach is that only the injected compartment is sampled, but we hope to obtain data on compartment 3 by examining the output of radioactivity in milk and respiratory $\mathrm{CO}_{2}$. Further insights into the validity of the model will be sought by the simultaneous use of ${ }^{3} \mathrm{H}$-labelled and ${ }^{14}$ C-labelled glucose.

\section{REFERENCES}

Berman, M. (1963). Ann. N.Y, Acad. Sci. 108, 182.

Berman, M. \& Schoenfield, R. (1956). F. appl. Phys. 27, г361.

Berman, M. \& Weiss, M. F. (1967). SAAM Manual, USPHS Publication No. 17o3. Washington, DC: US Government Printing Office.

Bickerstaffe, R., Annison, E. F. \& Linzell, J. L. (I974). F. agric. Sci., Camb. (In the Press.)

Infield, J. M., Robb, J., Horsfield, S. \& Annison, E. F. (1974). Publs Eur. Ass. Anim. Prod. (In the Press.)

Judson, G. J. \& I Jeng, R. A. (1968). Proc. Aust. Soc. Anim. Prod. 7, 354.

Julius, R. S. (1972). Comput. Bioned. Res. 5, 473.

Kronfeld, D. S., Ramberg, C. F. Jr \& Shames, D. M. (r97r). Am. F. Physiol. 220, 886.

Leng, R. A. (1970). Adv. vet. Sci. 14, 209.

Skinner, S. M., Clark, R. E., Baker, N. \& Shipley, R. A. (r959). Am. 7. Physiol. r96, 238.

Steele, R. (1964). Fedn Proc. Fedn Am. Socs exp. Biol. 23, 671.

Steele, R., Wall, J. S., de Bodo, R. C. \& Altszuler, N. (1956). Am. F. Physiol. I87, 5. 